
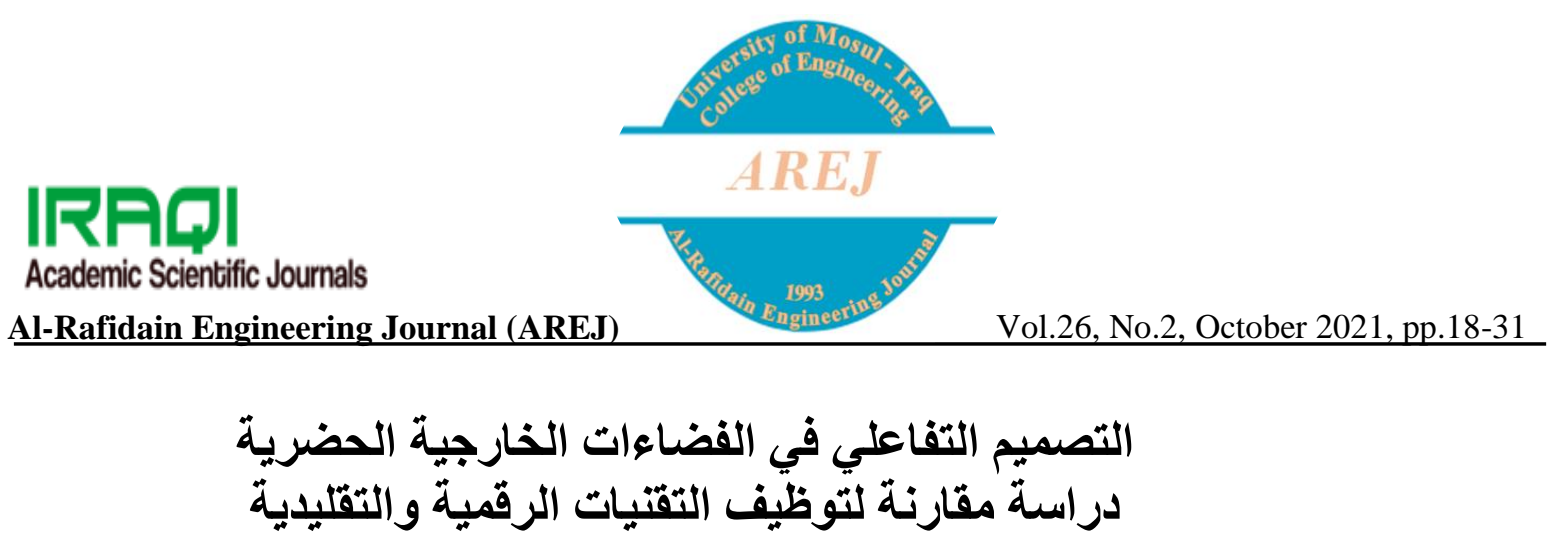

\section{د. احمد يوسف العمري}

ahmed.alomary@uomosul.edu.iq

\section{سهرى عماد الدبوني}

suha.enp122@student.uomosul.edu.iq

جامعة الموصل - كلية الهندسة ـ قسم هندسة العمارة

ناريخ القبول: 26/3/2021
تاريخ الاستلام: 23/1/2021

الخلاصة

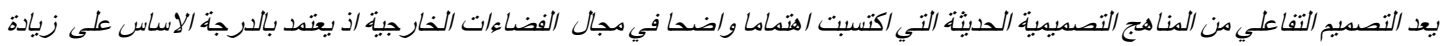

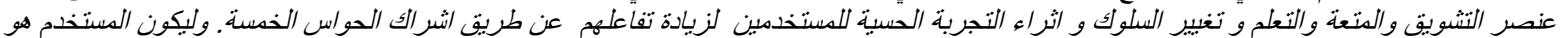

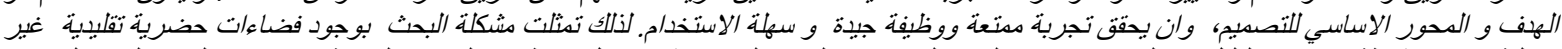

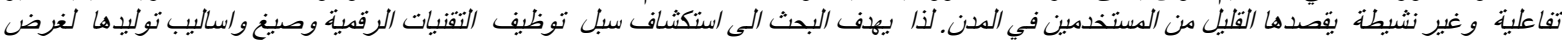

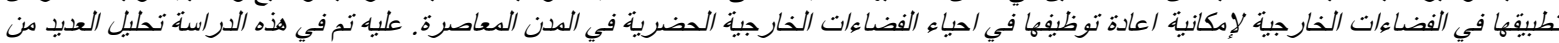

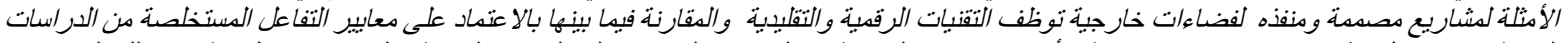

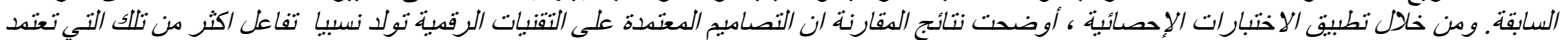

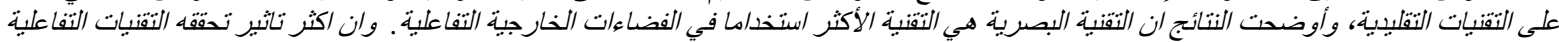
على المتلقي يتمثل بإثارة الاهتمام والفضول .

الكلمات الدالة:

التفاعل ، الفضاءات الخارجية الحسية ، الفضاءات الخارجية الحضرية، التصميم التفاعلي، التقنيات التفاعلبة .

This is an open access article under the CC BY 4.0 license (http://creativecommons.org/licenses/by/4.0/). https://rengj.mosuljournals.com

التي يمكن تحسها عن طريق اللمس. والروائح التي تحفز حاسة

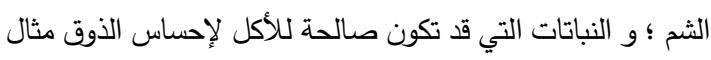
على ذلك "الحدائق الحسية" والتي هي جزء من البيئات العلاجية ،

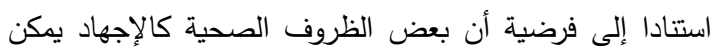
تحسينها من قبل المشاركة الحسية المناسبة وكل هذا يعمل كأجزاء من لوحة الفضاءات الخارجية الحسية. ان توظيف التقنيات

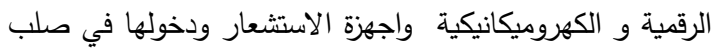

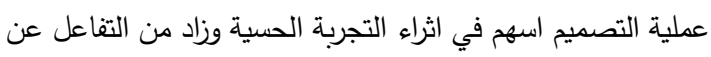
طريق الحواس الخمسة و خصوصا حاسة البصر و السمع واللمس،

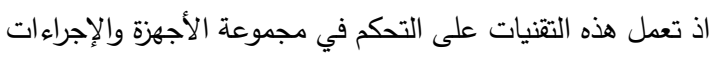
وتحويلها إلى اصوات رقمية ومرئية وعن طريق مواقع الويب يتم

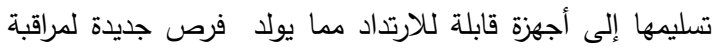
النظم البيئية و تمكين الجوانب الديناميكية لتجربة الفضاءات

الخارجية[2].

اما فيما يتعلق بالتقنيات الغير رقمية التقليدية فان المشهد التفاعلي

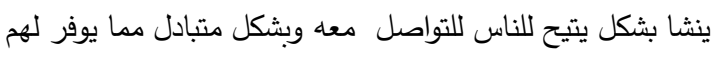

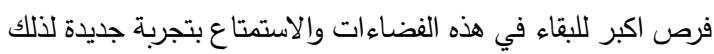

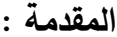

تعددت التقنيات التفاعلية التي طرحتها الدراسات السابقة وفي هذا الجانب يمكننا ان نلمس التطور الكبير الذي احدثته الثورة الرقمية

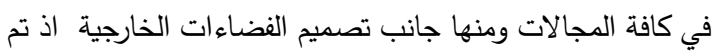

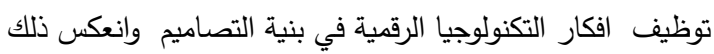
على الفضاءات الخارجية واصبحت ذذات سمة جديدة زاد فيها عنصر التثويق والمتعة والتعلم و تغيير السلوك وجوانب اخرى واصى كثيرة، اذ اقترح (dekel) ثلاث مشاريع تفاعلية تطبق في وني الفضاءات العامة وذلك لتحسين اشكال التفاعل الممكنة من خلال

شلالات موسيقية ، كرسي موسيقي ، مساطب حميمة [1] . و عرفت الفضاءات الخارجية الحسية(Sensory Landscapes) بانها البيئات التي تم تصميمها خصيصًا لتحفيز الحواس، فبالاضافة

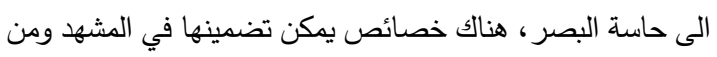

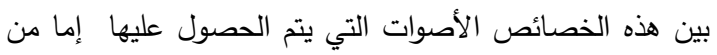

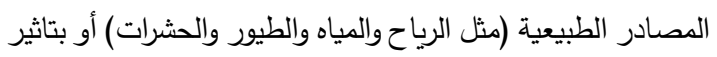
أكثر عن طريق التكنولوجيا (مكبرات الصوت ، أجهزة الإنذار) لإشراك الحس السمعي. كذلك القوام ، والأنماط ، وخصائص المواد 
:(Abdel-Aziz, Abdel-Salam, \& El- دراسة

Sayad, 2016)

تتناول الدراســة اثر انتثــار تكنولوجيا المعلومات والاتصــالات (ICTs)

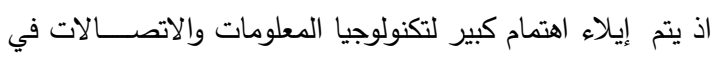

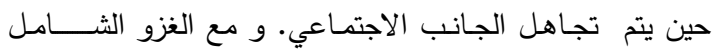

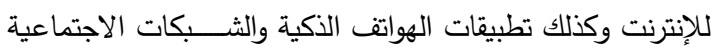
الرقمية، يصبح الناس أكثر ارتباطًا اجتماعيًا من خلال المساحات ولات

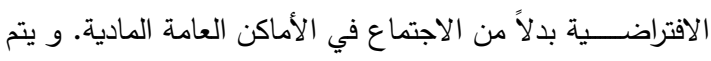
تصـنيف تكنولوجيا المعلومات والاتصـالات في هذه الدراسـة إلى لى أربعة عناصـر هي كما يلي: شـبكات Wi-Fi وواجهات الوسـائط التفاعلية الرقمية (digital interactive facades media) displays) (interactive public والعروض العامة التفاعلية وتطبيقـات الهواتف الذكـية في الأماكن العامة

(smartphones' applications in public space) تلعب هذه العناصـر أدوارًا رئيسية في الفضـاء العام ضـمن خمسـة

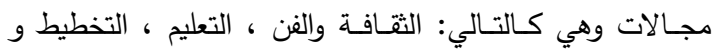

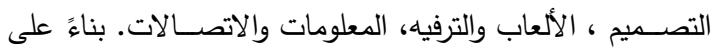
هذا التصـــنيف ، هناك العديد من الأمثلة والمقترحات لتكنولوجيا

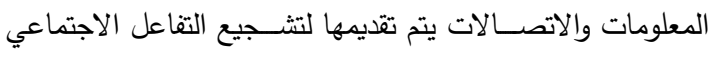

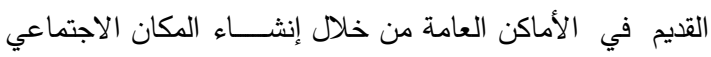
العام الجديد لهذا العصر الرقمي[6]. 4-Au \& Lee, 2017)

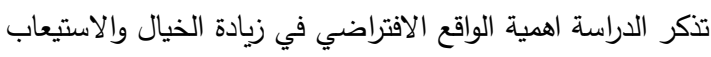

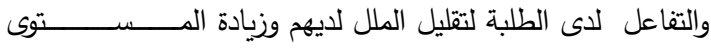
التعـليـمي ويـــد الواقــع الافتراضــي وسيلة تــــــاعليــة تشاركية

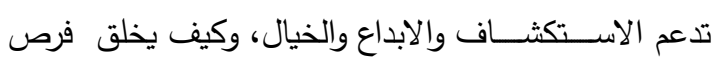
جديدة تدعم المتعلمين اذ يعد الواقع الافتراضــــي أداة للتعلم

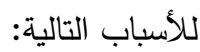
1 - أنها تجمع الطلاب الصغار بثكل تجربي وهي الطريقة التي يفضلونها 2- يحتاج نظامنا التعليمي الحالي إلى تجارب جذابة ومثيرة تقود التعلم الناجح. 3- يمكن أن توفر تقنية إمكانية تعريض الطلاب للعوالم والأشخاص الذين يتعذر الوصول إليهم عادة [7].

5 (Allcoat \& von Mühlenen, 2018) دراستة تم في هذه الدراسة المقارنة بين ثلاثة طرق تعليمية وهي : التقليدية

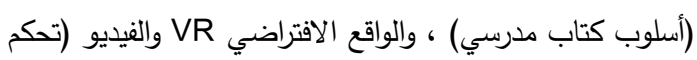

يجب ان يكون المشهد سريع الاستجابة ، نشطا وحساسا ، وفي حوار دائم معنا كمستخدمين وكسكان • وتذكر (huo) موقفا جديدا

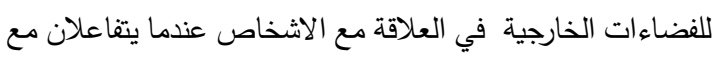
بعضهما البعض وتتتبا بان التجربة الجديدة التي يمكن تقديمها للناس تشمل تحول الفضاء من سلبي الى نشط ومن غير مرئي

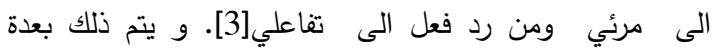

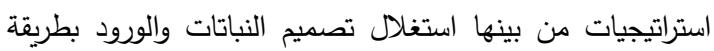
تفاعلية جميلة وباشكال جذابة تعمل على جذب الاشخاص والتجمع حولها وهو مايسمى بفن التشكيل او فن توباري Topiaire art. وكذلك استخدام طرق جديدة في تصاميم فضاءات ملاعب الاطفال

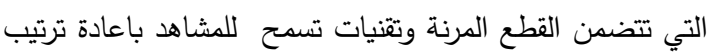

الفضاء حسب ذوقه إضافة الى تقنيات الخداع البصري لـالخ. الستائر ذات الانفتاح الذاتي الذي كان رد فعل لحركة المشاة و لفئه الظروف الجوية لتوفير بيئة مريحة في جميع الظروف[4].

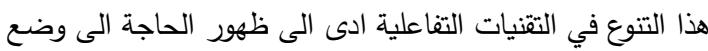
اسس للمقارنة بين الاساليب الرقمية وغير الرقمية التقليدية للافكار .

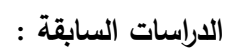

أ-الدراسات المتعلقة بالتقنيات الرقمية

1 (Schnabel \& Karakiewicz, 2007) تم خلال هذه الدراسة تقديم ثلاث مقترحات تفاعلية يشمل المقترح الاول التفاعل السمعي لغرض التخلص من الضوضاء من خلال عمل جدار تفاعلي يحول الضوضاء الى موسيقى وبذلك يكون قد حقق تفاعل وظيفي وترفيهي ، المقترح الثاني شمل تفاعل بصري

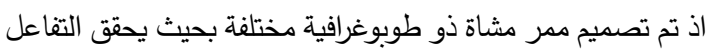
في حركة وتجارب وتفاعلات المشاة مع الطوبوغرافية اذ انهم سابقا

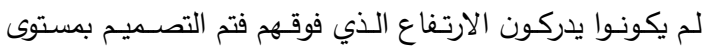
الثارع، المقترح الثالث يثـل تفاعل بيئي اذ تم إنثـاء أجهزة

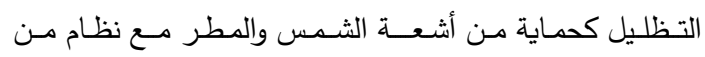
2-2 دراسة (Grønbæk, 2012)

لقد تم خلال هذه الدراسة تحويل فضاء ميت الى فضاء حيوي

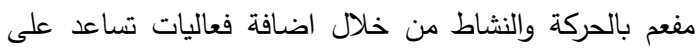

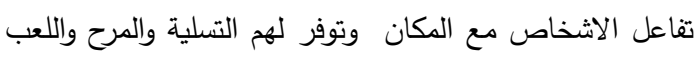

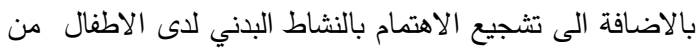

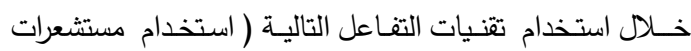
للحركة مدمجة في مقاعد التأرجح ومصابيح LED ونظام مكبر

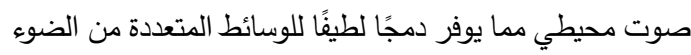
والصوت وبذلك تكون هذه الدراسة قد استخدمت تفاعل حركي بصري وسمعي )[5]. 


\section{ب - الدراسات المتعلقة بالتقنيات التقليدية}

:

تذكر الدراسة ان الجمالية الحركية تقاس بمستوى المتعة الناتجه

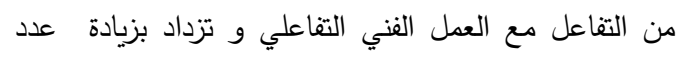
التجارب الحركية للمتلقي في العمل التفاعلي. وقد استتنجت

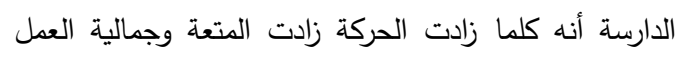
التفاعلي و الخبرة لدى المتلقي و العكس صحيح. ويذكر بان

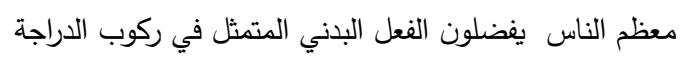
على الفعل البدني المتمثل في صنع حركات المعصم الصغيرة باستخدام فأرة الكمبيوتر ، وبالتالي ، سوف تحصل فلى على على درجة

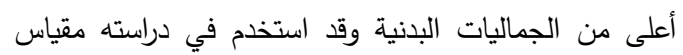
Physical Aesthetics Pleasantness ) PAPS (Score لقياس جودة التجربة الحركية عند استخدام وسيط

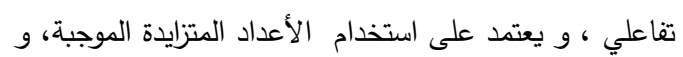

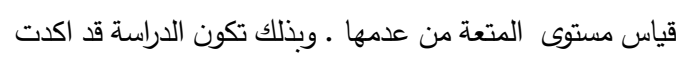
على أهمية جعل التصميم محفز للحركة كجعل حيز لممارسة فئسة رياضة معينة تشجع على النشاط البدني مما يحقق وظيفة ترفيهية وصحية معا ويمكن ان يتم ذلك عن طريق عدة تقنيات

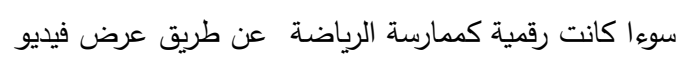

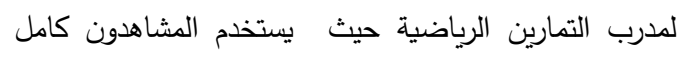
الجسم للتفاعل مع العرض ويقف مدرب التمارين الرياضية في لئري

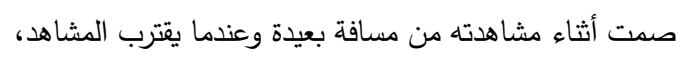
تبدأ الموسيقى ويبدأ المدرب في التمرين. وفي حال توقف أحد منداء

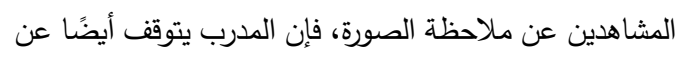
مدارسة التمارين وسـوف تتوقف الموسيقى او ميكانيكية او

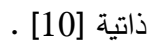

$$
\text { 8- دراسة (علي، 2013) : داتية }
$$

لقد تم خلال هذه الدراسة توضيح الهمية الاضاءة في انشاء

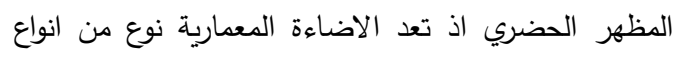

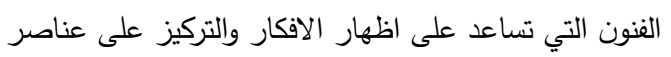

معينة لتجعل المشهد مثيرا للاهتمام وتساعد الاضاءة على تغير

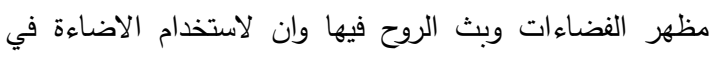
الفضاءات الخارجية فوائد متعددة تشمل فوائد وظيفية واخرى جمالية، اما الفوائد الوظيفية فتشمل تامين الاضاءة ونواحي الامان

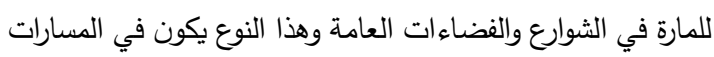
والمداخل والتقاطعات، وقد ذكرت الدراسة ان للاضاءة فوائد عديدة فهي تحدد كل من المسار والكتل والاشكال وتستخدم كمؤثر لاظهار الملمس وكمؤثر لتغير اللون اما الفائدة الاخرى فهي فوائد
سلبي) واستخدم نفس النص والنموذج ثلاثي الأبعاد لجميع الظروف وهو عبارة عن خلية نباتية .

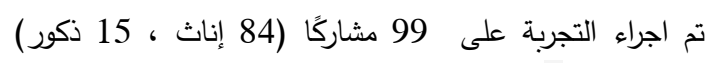
وجميعهم من طلاب علم النفس في السنة الأولى في جامعة Warwick التعليمي للمشاركين في الظروف التقليدية وظروف الواقع

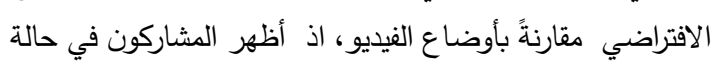

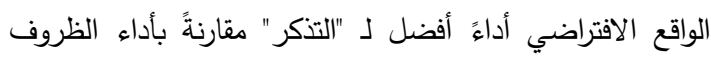

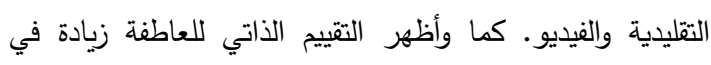
المشاعر الإيجابية وانخفاض في المشاعر السلبية لحالة الواقع الافتراضي. على العكس، كان هناك انخفاض في المشاعر الإيجابية في كل من الظروف التقليدية والفيديو • ووجد مقياس تقييم

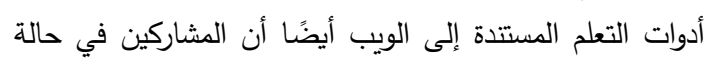

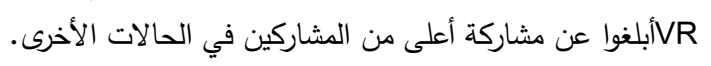

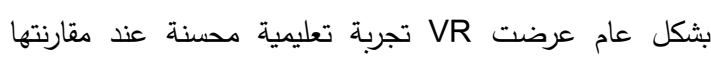

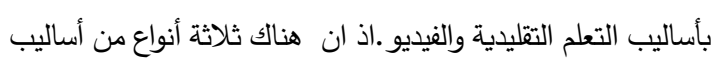

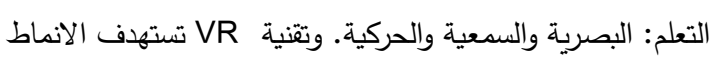

الثلاثة في تطبيق واحد [8].

Crowell, Sayis, Bravo, \& )

:(Paramithiotti, 2018

تتاولت الدراسة اهمية استخدام تكنولوجيا المعلومات والاتصالات لإنثاء بيئة تعاونية بين الأطفال الذين يعـانون من مرض التوحد

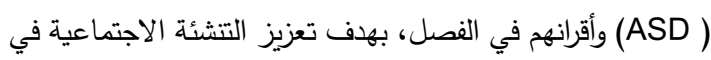
البيئة الصفية من أجل تسهيل التواصل الاجتماعي و توفير موارد

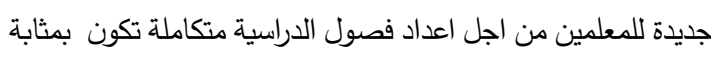
دعم لزيادة الاندماج خلال أنشطة اللعب. اذ تم انثاء أرضية تفاعلية يتحرك فيها كامل الجسم لاكتشاف مدى التفاعل و أجريت تجربتين

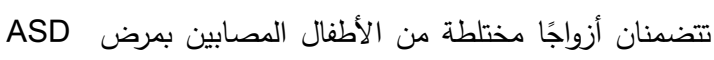

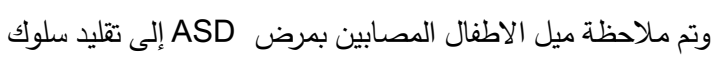
الاطفال الآخرين من أجل فهم اللعبة وبهذا يتحقق التفاعل

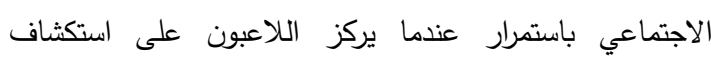
الرسومات التوليدية. توضح الدراسة اهمية التفاعل على النواحي

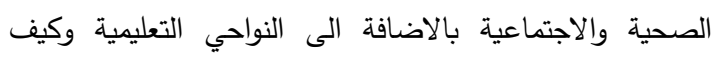
ساعدت الارضيات التفاعلية الحساسة للحركة من زيادة التفاعل لدى الاطفال المصابين بالتوحد وكيف زادت من التفاعل الاجتماعي

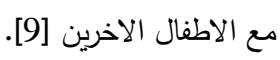


ومحفزة حيث يمكن للطلاب الدراسة والاستكثاف، بغض النظر عن احتياجاتهم التعليمية. ان غياب البيئة الآمنة و الديناميكية والتعاونية يسبب مشاكل صحية وقضايا اجتماعية وسلوكية و ذكر الباحث ان للتواصل مع الطبيعة تأثير إيجابي على صحة الطلاب حيث

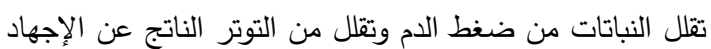
وتقلل من الغضب والخوف، حيث وجد الباحثون زيادة في أعراض عن عن الاض

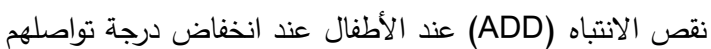
مع المناطق الخضراء[13]. 12-دراسة (شحاتة، محمد... وآخرون، 2018): ذكرت الدراسة احد الأساليب التفاعلية غير الرقمية في الفضادات الخارجية الا وهي التفاعل عن طريق التصميم الاعلاني : اذ تكون هناك علاقة متبادلة بين المرسل والمتلقي. وذكرت اساليب عديدة لتحقيق التفاعل منها استغلال عناصر البيئة باستخدام عناصر الفضاء لغرض الاعلان كاستخدام محطات الحافلات لعرض الاعلان ممايجعل الاعلان يدوم لفترة أطول. و يذكر الباحثون وسيلة أخرى للتفاعل الا وهي الألعاب اذ ان المتعة لاتتحصر بعمر معين ولذلك فان معظم الاشخاص يميلون للمرح وتم خلال هذه الدراسة انشاء مقترحين للاعلانات الاول : اعلان dove تحت شعار (dove is in the air ) سنة 2017 والثاني : نموذج للاثاث الذكي لشركة (IKEA) تحت شعار حرك الاثاث واكتثف ذكاء التصميم سنة 2018 تم الاعلان في مصر والذي يهدف الى تحفيز المتلقي للتفاعل مع التصميم ـ ان الاسلوب المستخدم وضع المتلقي تحت التجريب ليشارك في الاعلان [14]. مشكلة البحث : - مثي

وجود فضاءات حضرية تقليدية في المدن غير تفاعلية وغير نشيطة

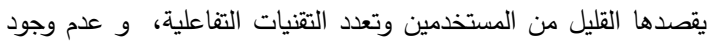
اطار نظري لتحقيق التفاعل والنشاط لمستخدمي الفضاءات الخارجية التقليدية .

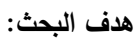
استكثاف توظيف التتنيات التفاعلية الرقمية و غير الرقمية التقليدية

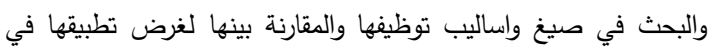
الفضاءات الخارجية لاككانية اعادة احياء الفضاءات الخارجية الحضرية الغير فعالة في المدن المعاصرة. المفردات المستخلصة :

تم ومن خلال تحليل الدراسات السابقة انتخــاب مفردتين رئيسيتين الأولى طبيعة التقنيات المستخدمة في التصميم واسلوب توظيفهاو المفردة الثانية
جمالية زخرفية وتعطي انفعالات حسية بصرية و نفسية كالبهجة والسرور و فوائد تصميمية ( التاطير، التوجيه، الوضوحية، الاحتواء). ولم تتطرق الدراسة الى امكانية استخدام الاضاءة كوسيلة للاعلان او الاحتفال مما يزيد من التفاعل. كما ان الدراسة اقتصرت على الجوانب غير الرقمية للاضاءة ولم تذكر العروض الليزرية

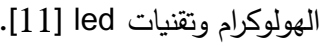

9enkatesha, 2014) دراسة

تشمل هذه الدراسة اقتراح استخدام الفضاءات الخارجية لمساعدة طلاب المرحلة الثانوية في التقليل من الضغط النفسي كما تتاقش كيفية تصميمها وفقا لاحتياجات ورغبات كل فئة عمرية. ان تصميم الفضاءات الخارجية مهم للمدراس الثانوية لان الطلاب في هذه المرحلة معرضين للانتحار والمخدرات لذلك هم بحاجة الى بيئات محمية و مسيطر عليها لتساعدهم على التخلص من التوتر والاجهاد. طبقت هذه الدراسة على ثلاث مدارس ثانوية عامة في مئي ولاية إلينوي الوسطى لفحص اهتمامات الطلاب في تكوينات المناظر الطبيعية الموجودة في حرم المدارس الثانوية[12]. :(huo, 2016) 10

تم خلال هذه الدراسة مناقثة الفضاءات الخارجية التفاعلية والتي تعمل على تقديم وظيفة جديدة للفضاءات الخارجية وهي ان تكون مساحة يتفاعل فيها الناس مع المنظر ومع بعضهم البعض ونوفر

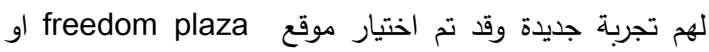
مايعرف باسم Western plaza كموقع للدراسة في واشنطن. حددت هذه الدراسة اهم الميزات التي تجعل التصميم تفاعلي وكذلك اهم الطرق التي تساعد على التحفيز وجذب الانتباه ولقد تم تحليل العديد من الامثلة عن طريق الربط بين ميزات التصميم التفاعلي وطرق التحفيز • وعليه فان هذه الدراسة لم تذكر انواع التصاميم

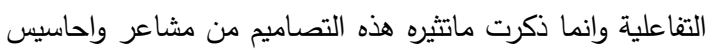

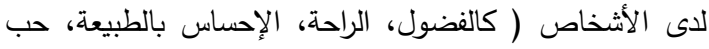

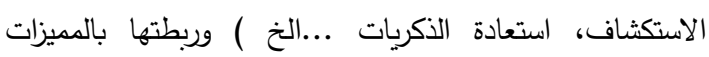
التصميمية ( الثكل، المقياس، استغلال المناطق السالبة، مرئي

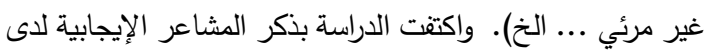
الأشخاص ولم تتطرق انه في بعض الأحيان ممكن للتصميم اثارة

بعض احاسيس الاضطراب،الخوف ،الرهبة ، القلق ...الخ) [3].

:11-دراسة (Elmaghraby \& Kenawy, 2016) تؤكد الدراسة على أهمية الفضاءات الخارجية للمساعدة في الحد من التوتر الذي يؤثر على الطلاب خلال يومهم الدراسي وزيادة الأداء الأكاديمي علاوة على أن الطلاب يستفيدون سلوكياً وبيئياً

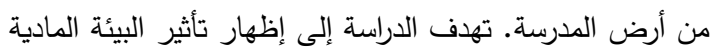
في الهواء الطلق في المدارس على الطلاب. ، وتوفير بيئات آمنة 
نوع التأثير التفاعلي الذي تحدثه التقنية للى المتلقي وتم اشتقاق المفردات

الثانوية وكما موضح في الجدول رقم (1)

\begin{tabular}{|c|c|c|c|c|}
\hline الترميز & & القيم الممكنة & المفردات الثانوية & المفردات الرئيسية \\
\hline 1 & & & تقنيات بصرية & \multirow{23}{*}{ توظيفهة التقنيات و اسلوب } \\
\hline \multirow{2}{*}{\multicolumn{3}{|c|}{$\begin{aligned} 1.1 \\
1.1 .1\end{aligned}$}} & \multirow{11}{*}{ تقنيات بصرية } & \\
\hline & \multicolumn{2}{|r|}{ اضصاءة } & & \\
\hline 1.1 .1 .1 & كمؤثر على لون العناصر & & & \\
\hline 1.1 .1 .2 & تحديد المسار & & & \\
\hline 1.1.1.3 & محدد للكتلة & & & \\
\hline 1.1 .1 .4 & محدد للملمس & & & \\
\hline 1.1.1.5 & وسيلة للاعلان و الاحتفال & & & \\
\hline 1.1 .1 .6 & عروض ليزرية (هولوكر ام) & & & \\
\hline 1.1 .1 .7 & led تقنيات & & & \\
\hline 1.1 .2 & & ايهامات بصرية & & \\
\hline 1.1 .3 & & الو اقع المعزز & & \\
\hline \multicolumn{3}{|l|}{1.2} & \multirow[t]{4}{*}{ تقنيات حركية } & \\
\hline 1.2 .1 & & ميكانيكية & & \\
\hline 1.2 .2 & & رقمية & & \\
\hline 1.2 .3 & & ذاتية & & \\
\hline \multicolumn{3}{|l|}{1.3} & \multirow[t]{3}{*}{ تقنيات سمعية } & \\
\hline 1.3 .1 & \multicolumn{2}{|c|}{ اصوات طبيعية كاصو ات الطيور و المياه } & & \\
\hline 1.3 .2 & \multicolumn{2}{|c|}{ اصوات اصطناعية كاصو ات الموسيقة } & & \\
\hline \multicolumn{3}{|c|}{ (l) } & \multirow[t]{3}{*}{ تقنيات لمسية } & \\
\hline 1.4 .1 & \multirow{2}{*}{\multicolumn{2}{|c|}{ شاثشات تفاعلية }} & & \\
\hline 1.4 .2 & & & & \\
\hline 1.5 & & & \multirow[t]{2}{*}{ تقنيات نحتية } & \\
\hline 2 & & & & \multirow{27}{*}{ في المتلقي تاثير التقنيات التفاعلية } \\
\hline \multicolumn{3}{|l|}{2.1} & \multirow[t]{26}{*}{ 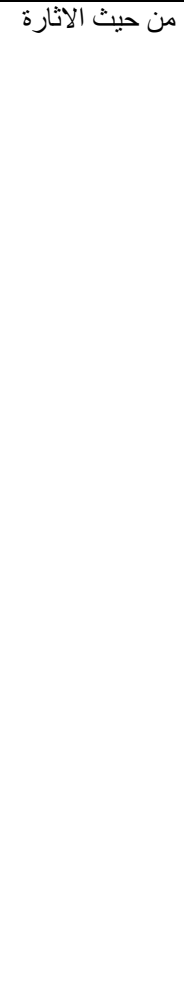 } & \\
\hline \multicolumn{3}{|r|}{ أثنارة الالهتمام } & & \\
\hline 2.1 .1 .1 & 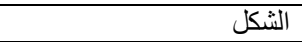 & و الفضول & & \\
\hline 2.1 .1 .2 & المقياس & & & \\
\hline 2.1 .1 .3 & مرئي ، غير مرئي & & & \\
\hline 2.1.1.4 & جمع بين وظيفتين & & & \\
\hline 2.1.1.5 & عكس الوظيفة & & & \\
\hline 2.1 .1 .6 & اضافة او تعزيز احساس & & & \\
\hline 2.1.1.7 & رد فعل متبادل & & & \\
\hline 2.1 .1 .8 & رد فعل مباشر & & & \\
\hline 2.1 .1 .9 & استغلال المناطق السالبة & & & \\
\hline 2.1 .2 & الفسح او الحيز المكاني & اثارة حب تملك حيز & & \\
\hline 2.1 .3 & & اثارة حب الاستكثاف & & \\
\hline 2.1 .3 .1 & التشويق & & & \\
\hline 2.1 .3 .2 & الالغاز & & & \\
\hline 2.1 .3 .3 & شكل غير مالوف & & & \\
\hline 2.1 .3 .4 & الغموض & & & \\
\hline 2.1 .4 & & اثارة الاحساس & & \\
\hline 2.1 .4 .1 & الصورة الذهنية & بالمكان و استعادة & & \\
\hline 2.1 .4 .2 & الفعالية & الذكريات & & \\
\hline 2.1.4.3 & المعنى & & & \\
\hline 2.1 .4 .5 & الصوت & & & \\
\hline 2.1 .4 .6 & روائح & & & \\
\hline 2.1 .4 .7 & رواية قصة او حدث معين & & & \\
\hline 2.1 .5 & & اثارة الحركة & & \\
\hline 2.1 .5 .1 & تحفيز الققز & & & \\
\hline
\end{tabular}




\begin{tabular}{|c|c|c|c|c|}
\hline 2.1 .5 .2 & تحفيز المشي او الركض & & & \\
\hline 2.1 .5 .3 & الوقوف المفاجىء & & & \\
\hline 2.1 .5 .4 & تحفيز التسلق & & & \\
\hline 2.1 .6 & & \multirow{5}{*}{ وزيادة التركيز الانتباه } & & \\
\hline 2.1 .6 .1 & اللون و الاضـاءة & & & \\
\hline 2.1 .6 .2 & المقياس & & & \\
\hline 2.1 .6 .3 & الصوت & & & \\
\hline 2.1 .6 .4 & الحركة المفاجئة & & & \\
\hline 2.2 & & & من حيث اضفاء الاحاسيس & \\
\hline 2.2 .1 & & \multirow{8}{*}{ 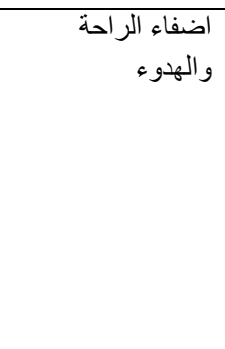 } & & \\
\hline 2.2 .1 .1 & الاشكال المالوفة & & & \\
\hline 2.2 .1 .2 & المقياس الانساني & & & \\
\hline 2.2 .1 .3 & الاحتو ائية & & & \\
\hline 2.2 .1 .4 & الاضاءة و الالو ان الهادئة & & & \\
\hline 2.2 .1 .5 & الصوت الهادئ & & & \\
\hline 2.2 .1 .6 & الوضوح & & & \\
\hline 2.2 .1 .7 & الطبيعة & & & \\
\hline 2.2 .2 & & \multirow[t]{7}{*}{ الثعور بالاضطراب } & & \\
\hline 2.2 .2 .1 & المقياس الضخم & & & \\
\hline 2.2 .2 .2 & عنصر مفاجئ & & & \\
\hline 2.2.2.3 & الاماكن الضيقة & & & \\
\hline 2.2 .2 .4 & الاضـاءة المتغيرة و الالوان الغامقة & & & \\
\hline 2.2 .2 .5 & الثكل الغريب & & & \\
\hline 2.2 .2 .6 & الأصو ات الصاخبة & & & \\
\hline
\end{tabular}

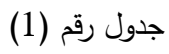

غير الرقمية) وذلك تبعا لاهميتها وتنوع وحداثة التقنيات التي

استخدمها غيرا

بالاثاث التفاعلي. وبذلك يكون العدد الكلي عشرون مثال (عشرة مشاريع

رقمية وعشرة تقليدية غير رقمية ) والجدول رقم (2) يوضح أسماء المشاريع

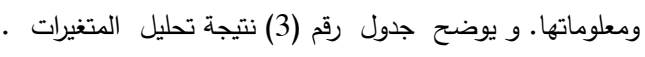

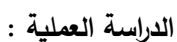
1- اختيار العينات : تم انتخاب 20 مشروع من المشـاريع المعماريةالعالمية (10تستخدم تقنيات رقمية و10 تستخدم تقنيات تقليدية المصممون فيها وبالاعتماد على مراجع تحوي معلومات كاملة مع مراعاة حداثة الأمثلة لتكون محصورة بالفترة الزمنية بين 2000-2020 2- تحليل العينات : تم انتخاب 20 مشروعا مصمم ومنفذ لفضاءات خارجية عالمية توظف التقنيات التفاعلية ـ وتم تقسيمها الى اربع مجاميع

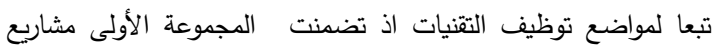

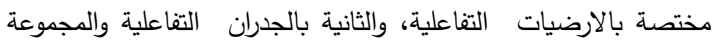

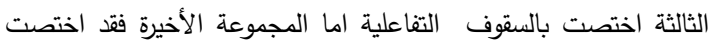

\begin{tabular}{|c|c|c|c|c|c|}
\hline صور المثال & الفكرة التصميمية & المصمح & والسمة المثال & والرقم & المجمو عة \\
\hline 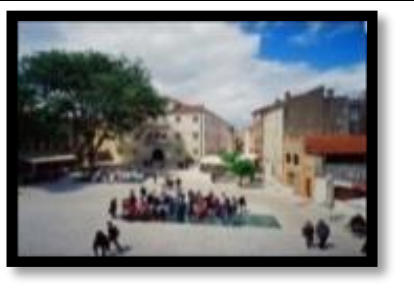 & 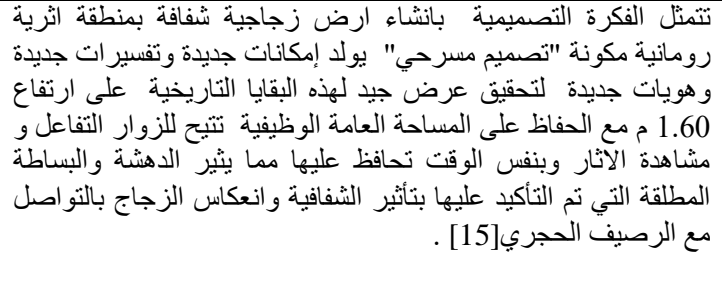 & $\begin{array}{r}\text { Kostrencic - } \\
\text { Krebel }\end{array}$ & $\begin{array}{r}\text { ساحة } \\
\text { Peter } \\
\text { Zorani } \\
\text { and } \\
\text { Budime } \\
2013\end{array}$ & 1- تقليدي & 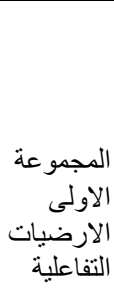 \\
\hline
\end{tabular}


24 Suha Imad Aldobouni: Interactive Design in Urban Outdoor Spaces .....

\begin{tabular}{|c|c|c|c|c|c|}
\hline صور المثال & الفكرة التصميمية & المصمح & والسم المثال & والرقم & المجمو عة \\
\hline 5 & 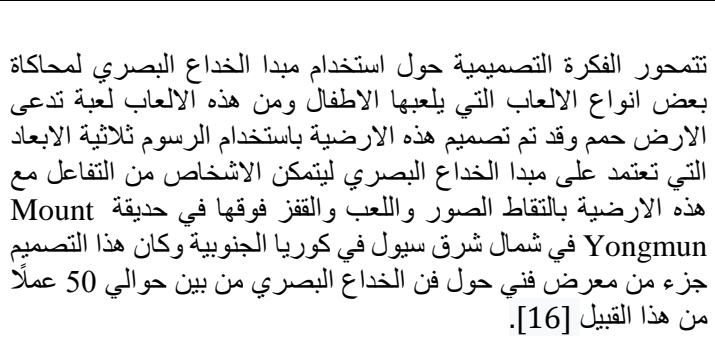 & EPA & أرضية & 2- تقليدي & \\
\hline 한 & 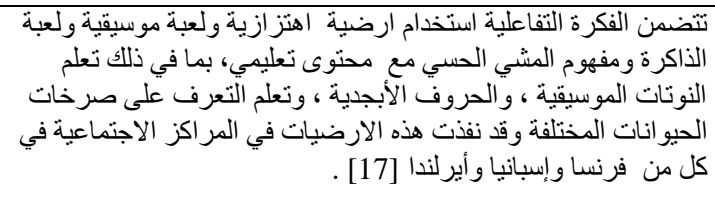 & شركة & تعليمية & رقمي & \\
\hline Ex & 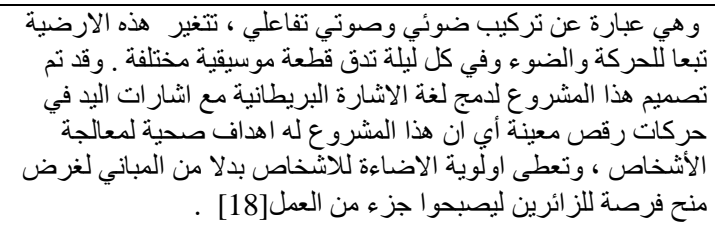 & $\begin{array}{l}\text { kit ويت مونكمان } \\
\text { Monkman } \\
\text { وتوم ويكسلر Womler }\end{array}$ & $\begin{array}{r}\text { Guildhal } \\
1 \\
\text { ارضية } \\
2008\end{array}$ & رقمي & \\
\hline & 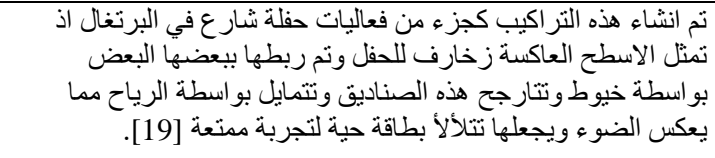 & $\begin{array}{r}\text { فيليب فروس } \\
\text { هilipa Frois } \\
\text { Hugo Reis }\end{array}$ & الصنادية & 5-تقليدي & الثانية الثمو عة الثفاعلة \\
\hline 5 & 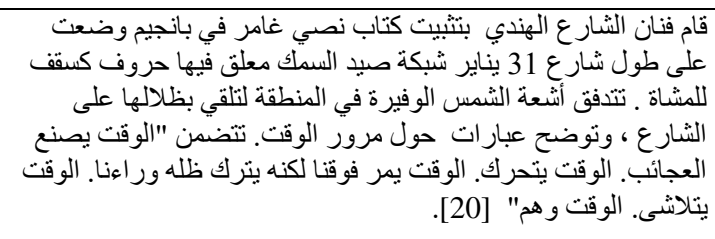 & DAKU & $\begin{array}{r}\text { النظرية } \\
\text { Theory } \\
2019\end{array}$ & 6 تقليدي & \\
\hline 1 & 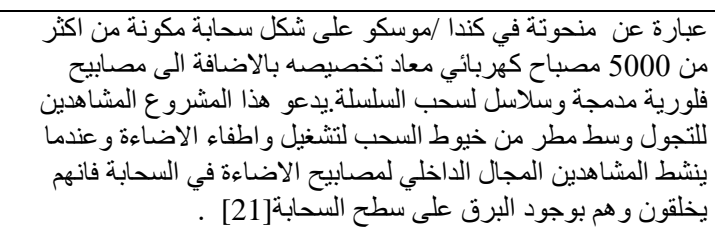 & البنانية الفينة كايتلاند & $\begin{array}{r}\text { سحابتلاند } \\
\text { Caitlind } \\
\text { Brown } \\
\text { Cloud } \\
2012\end{array}$ & 7-رقمي & \\
\hline 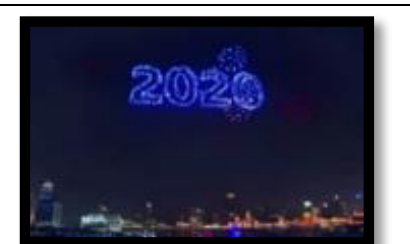 & 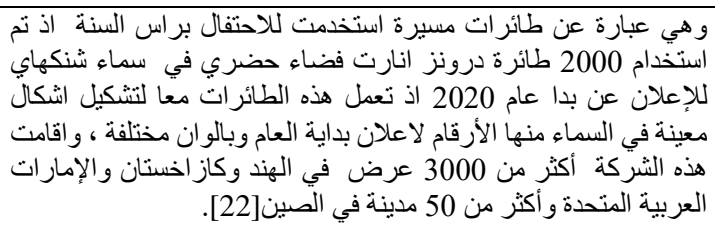 & $\begin{array}{r}\text { شركة } \\
\text { Shenzhen } \\
\text { DAMODA } \\
\text { Intelligent } \\
\text { Control } \\
\text { Technology }\end{array}$ & $\begin{array}{r}\text { Drons } \\
2020\end{array}$ & 8-رقمي & \\
\hline
\end{tabular}




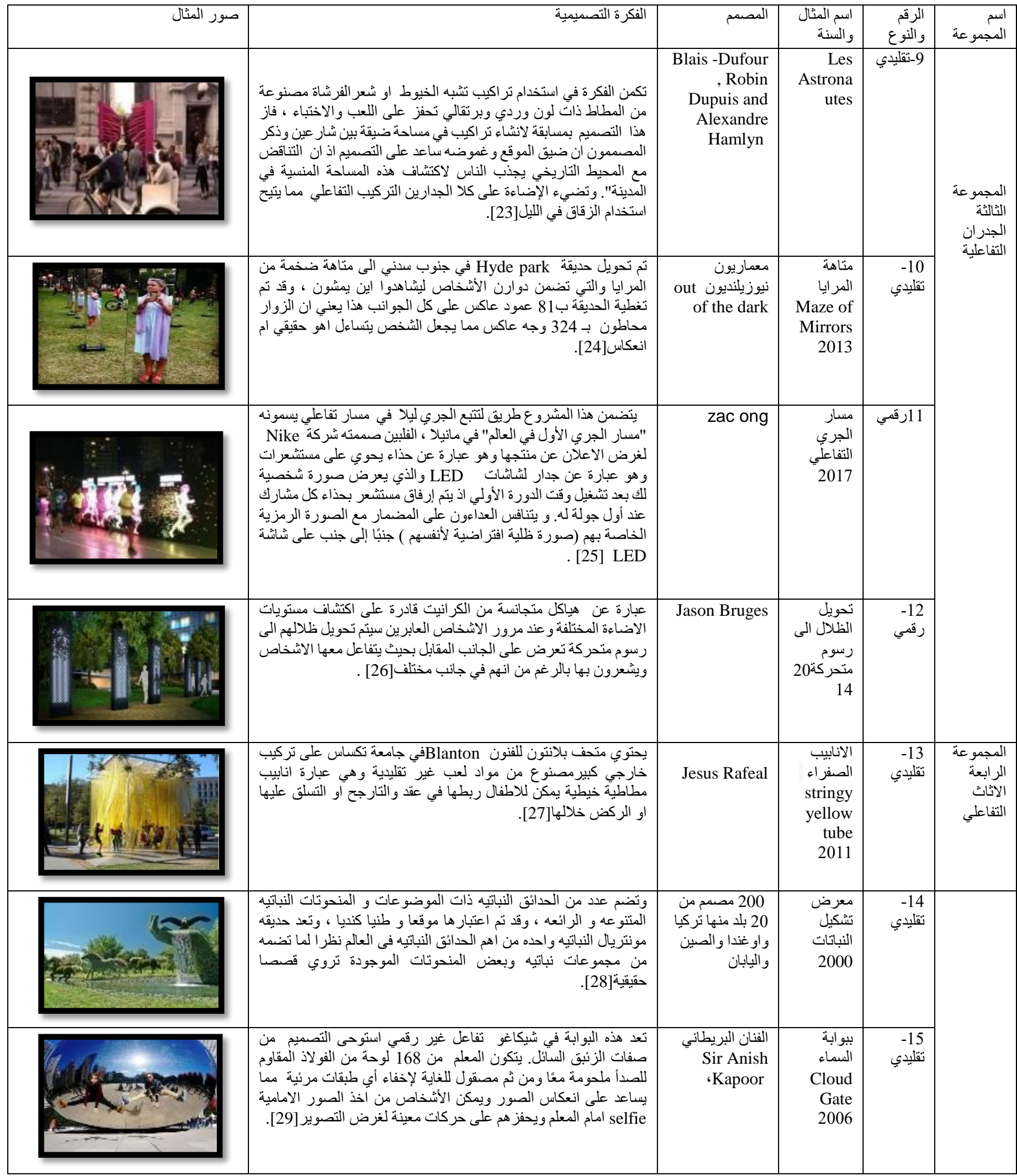


26 Suha Imad Aldobouni: Interactive Design in Urban Outdoor Spaces .....

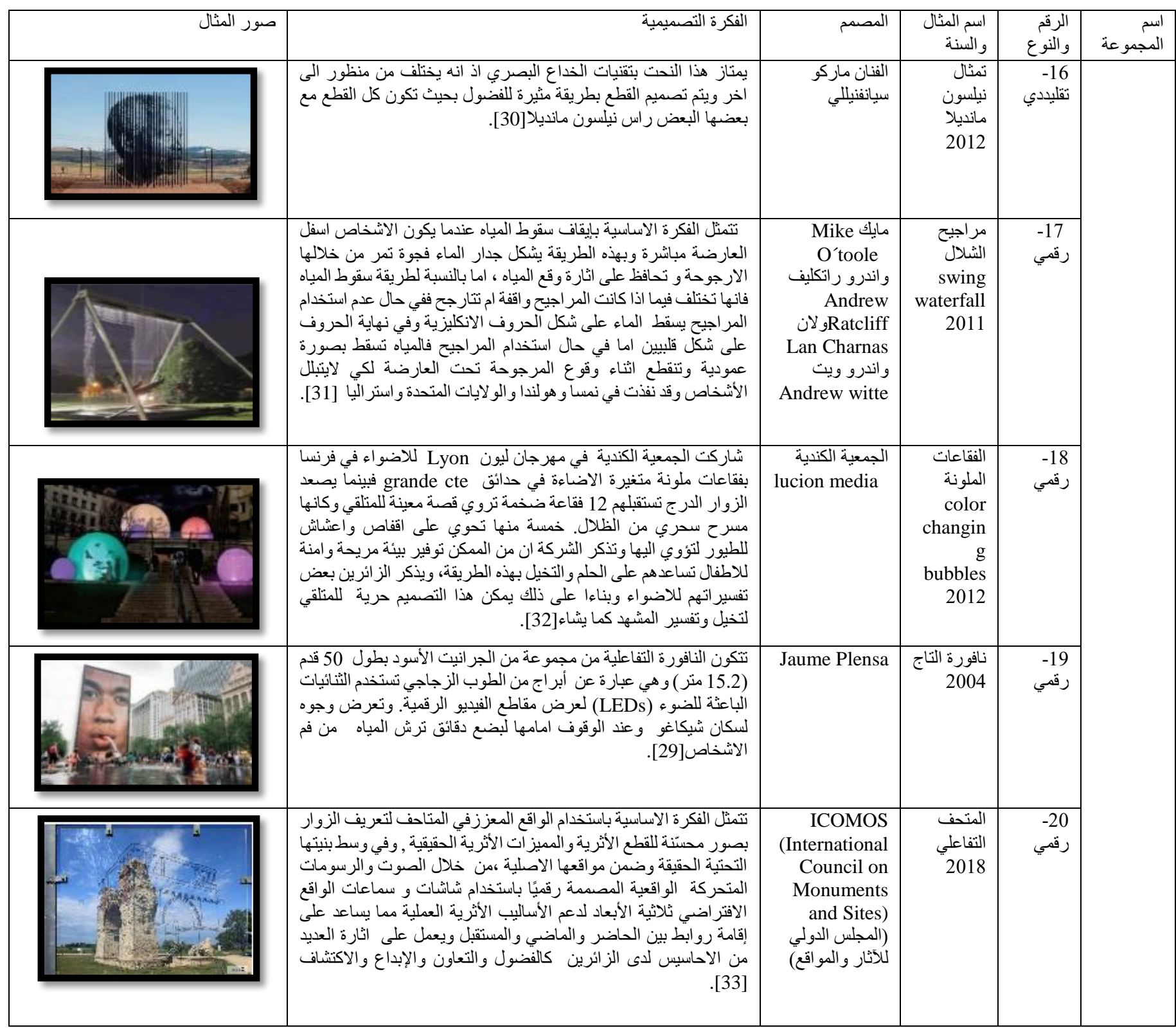

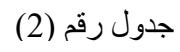




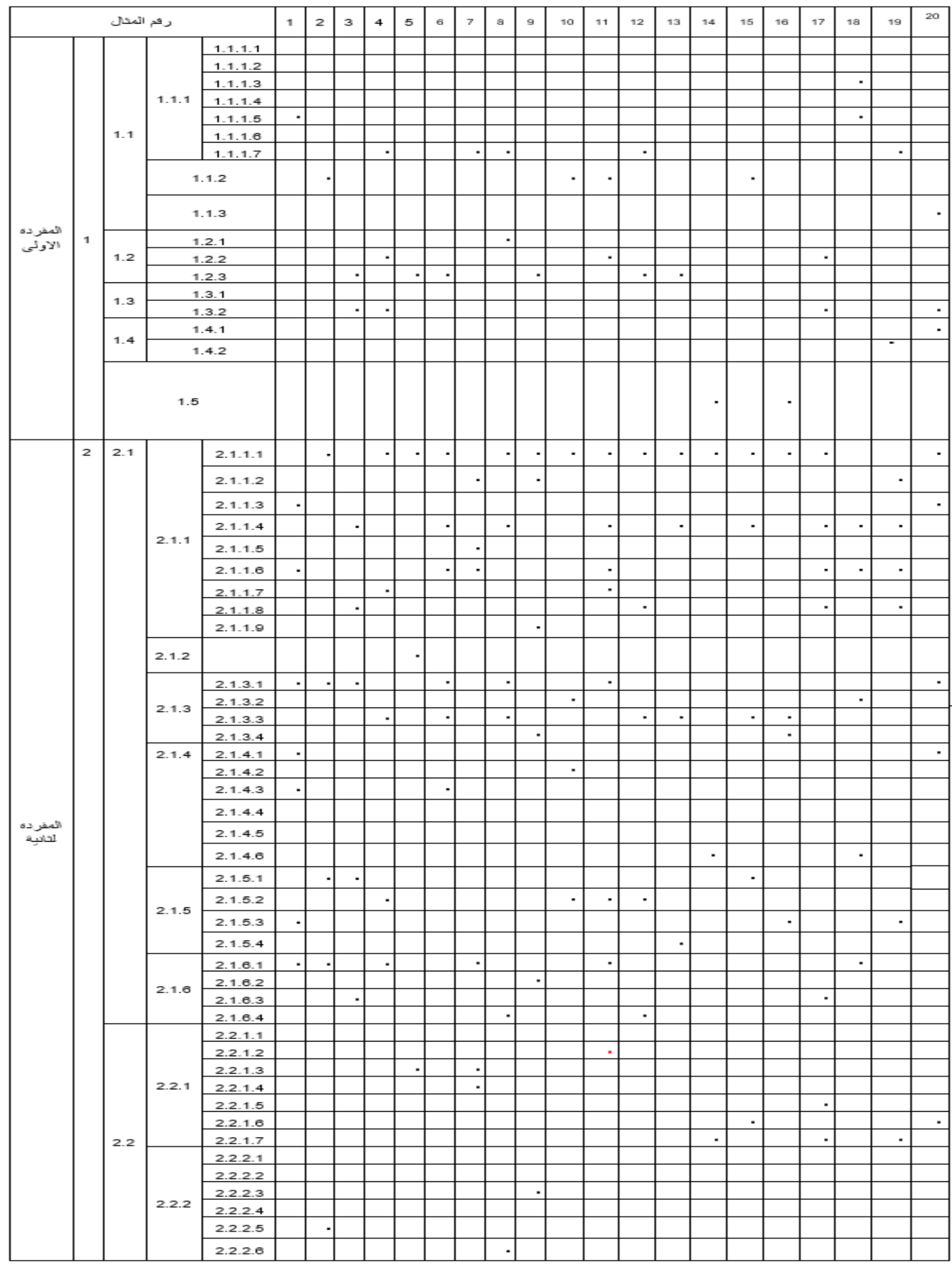

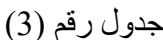




\begin{tabular}{|c|c|c|c|c|c|}
\hline تنقبك اللحثية & الثلتبكت الثيسية & المتكبت السيعة & بلتضبت الحرية & الثقلتبك تبمرية & رلفم الصدي \\
\hline 0 & 0 & 0 & 0 & 1 & 1 \\
\hline 0 & 0 & 0 & 0 & 1 & 2 \\
\hline 0 & 0 & 1 & 1 & 0 & 3 \\
\hline 0 & 0 & 1 & 1 & 1 & 4 \\
\hline 0 & 0 & 0 & 1 & 0 & 5 \\
\hline 0 & 0 & 0 & 1 & 0 & 6 \\
\hline 0 & 0 & 0 & 0 & 1 & 7 \\
\hline 0 & 0 & 0 & 1 & 1 & 8 \\
\hline 0 & 0 & 0 & 1 & 0 & 9 \\
\hline 0 & 0 & 0 & 0 & 1 & 10 \\
\hline 0 & 0 & 0 & 1 & 1 & 11 \\
\hline 0 & 0 & 0 & 1 & 1 & 12 \\
\hline 0 & 0 & 0 & 1 & 0 & 13 \\
\hline 1 & 0 & 0 & 0 & 0 & 14 \\
\hline 0 & 0 & 0 & 0 & 1 & 15 \\
\hline 1 & 0 & 0 & 0 & 0 & 16 \\
\hline 0 & 0 & 1 & 1 & 0 & 17 \\
\hline 0 & 0 & 0 & 0 & 2 & 18 \\
\hline 0 & 1 & 0 & 0 & 1 & 19 \\
\hline 0 & 1 & 1 & 0 & 1 & 20 \\
\hline 2 & 2 & 4 & 10 & 13 & التكرار \\
\hline 0.1 & 0.1 & 0.2 & 0.6 & 0.65 & التصبية \\
\hline
\end{tabular}

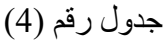

• نلاحظ في الثكل رقم (1) ان التقنية البصرية هي التقنية الأكثر استخداما في الأمثلة اذ انها استخدمت الفت الفت 13 من

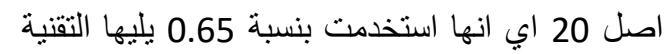

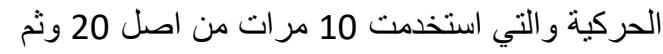
التقنية السمعية استخدمت 4 مرات واخيرا التقنيتين

اللمسية و النحتية استخدمت مرتين و بنسبة 0.1 التخدية

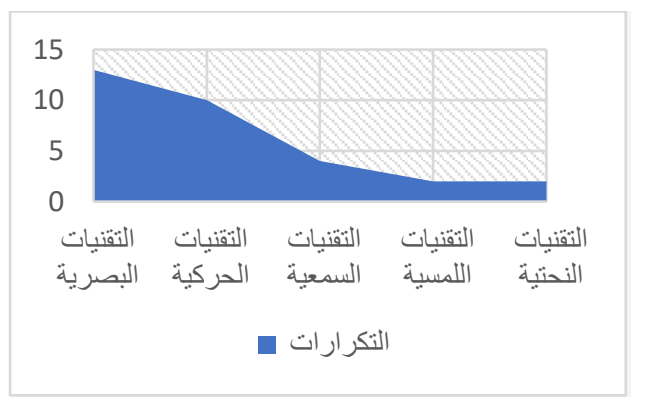

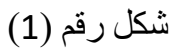

• اما بالنسبة للمعيار الثاني والذي يخص طبيعة نأثير الثقانتيات

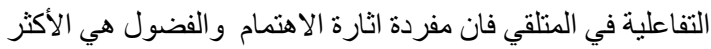

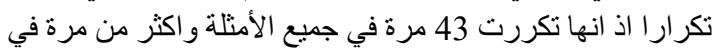

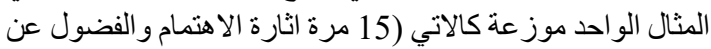

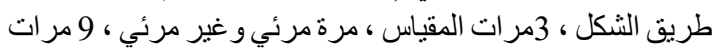

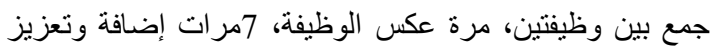

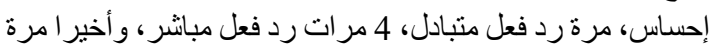

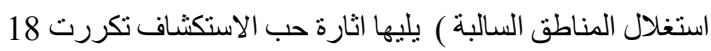

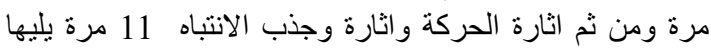

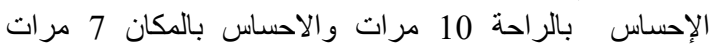

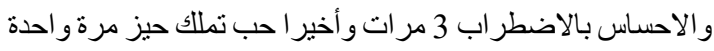

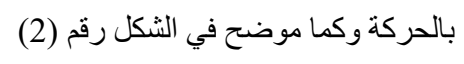

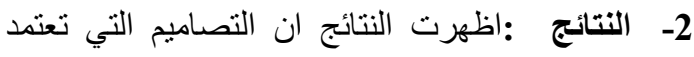
التقنيات الرقمية تعطي تفاعلا اكبر مقارنة بالتصاميم التئي

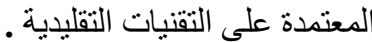

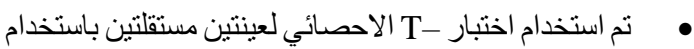

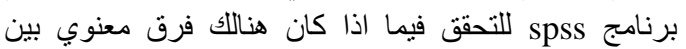
التصاميم التي تعتمد التقنيات الرقمية و التصاميم التقليدية

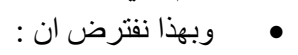

Ho ا

• وبعد تطبيق الاختبار اتضح ان هناك فرق معنوي بين

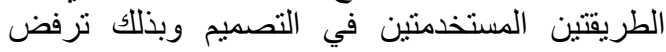

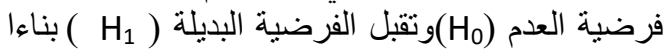
على القيم الناتجة من عملية التحليل الاحصائي ،اذ ان ان ان قيمة SIG.(2-TAILED)=0.001 وهي اقل من 0.05

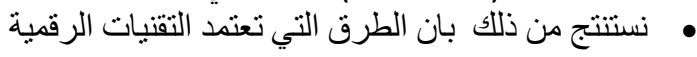
تحقق اكثر تفاعل من التقنيات التقليدية وذللك لان معدل التقنيات الرقمية ( Mean ) بساوي 7.9 وهو اكبر من

3عدل التقنيات التقليدية ( Mean ) و الذي يساوي 5.7 و

$$
\text { 3- الاستنتاجات : }
$$

تبين ان التصاميم المعتمدة على التقنيات الرقمية تعطي تفاعل اكبر

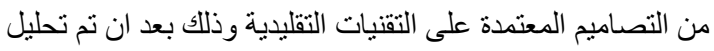

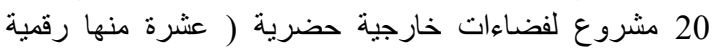
و عثرة تقليدية ) بالاعتماد على معيارين الأول التقنية المستخدمة

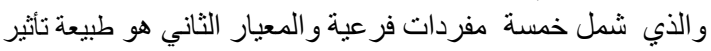

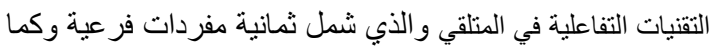

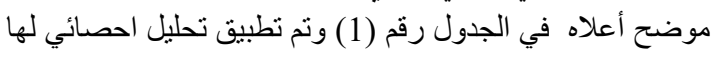

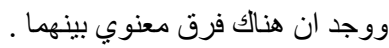
الاستتتاجات الخاصة بمفردات الاطار النظري

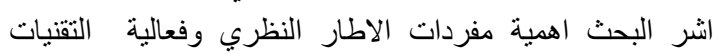
المستخدمة للمقارنة في التصاميم ( تقنيات بصرية ، تقنيات التيات حركية

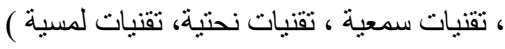
أثر البحث فعالية المقارنة في الجانب التفاعلي الممثل بردود الفعل التهل

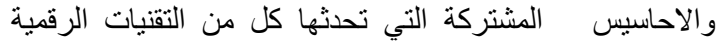

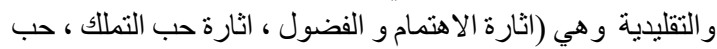

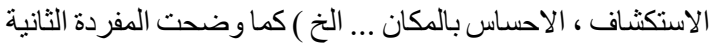

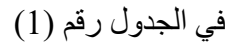

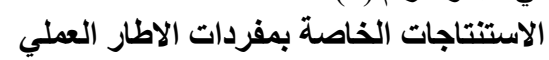

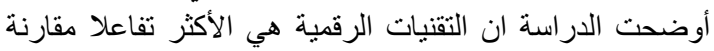

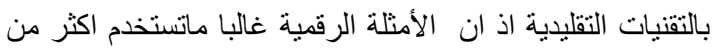

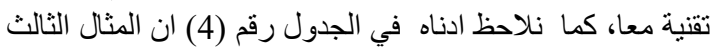

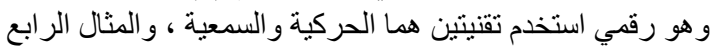

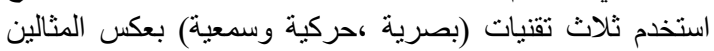

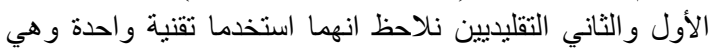
البصرية وكنلك الحال مع باقي الأمثلة . 


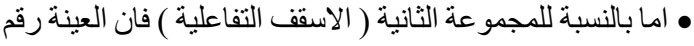

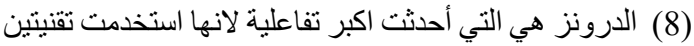

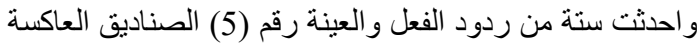

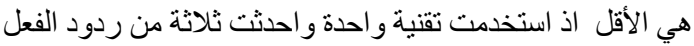

• وحصلت العينة رقم (11) مسار الجري ضمن المجمو عة الثالثة

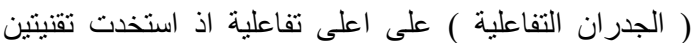

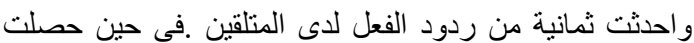

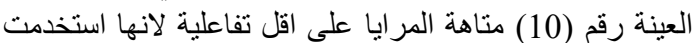

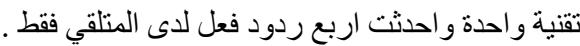

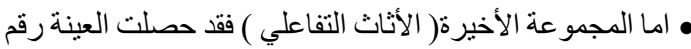

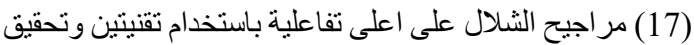

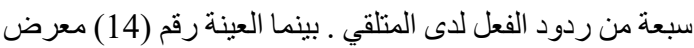
تشكيل النباتات هي الأقل تحقيقا للتفاعلية لانها استخدمت التهات تقنية

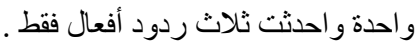

$$
\text { 4- التوصيات : }
$$

• أهمية ادخال التقتيات الرقمية في تصاميم الفضاءات

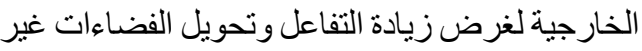

$$
\text { الفعالة الى مكان اجتماعي تفاعلي. }
$$

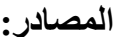

[1] A. Dekel, Y. Simon, et al. (2005). Adding playful interaction to public spaces. International Conference on Intelligent Technologies for Interactive Entertainment, Springer.

[2] E., S. M. (2018). "Sensor-y Landscapes: Sensors and Sensations in Interactive Cybernetic Landscapes." Journal of Digital Landscape Architecture: 96-106.

[3] huo, s. (2016). interactive landscape Rhoda island Rhoda island school of design master.

[4] M. A.Schnabel, and J. Karakiewicz (2007). "Rethinking parameters in urban design." International Journal of Architectural Computing 5(1): 83-98.

[5] G., K., Kortbek, Karen Johanne,Møller, Claus,Nielsen, Jesper, Stenfelt, Liselott (2012). Designing Playful Interactive Installations for Urban Environments-TheSwingScape Experience. Advances in Computer Entertainment.

[6] A., A. A., Abdel-Salam, H., \& El-Sayad, Z. (2016). The role of ICTs in creating the new social public place of the digital era. Alexandria Engineering Journal, 55(1), 487-493.

[7] H., E., \& Lee, J. J. (2017). Virtual reality in education: a tool for learning in the experience age. International Journal of Innovation in Education, 4(4), 215-226.

[8] A., D., \& von Mühlenen, A. (2018). Learning in virtual reality: Effects on performance, emotion and engagement. Research in Learning Technology, 26.

[9] C.1, C., Sayis, B., Bravo, A., \& Paramithiotti, A. (2018). GenPlay: Generative Playscape. Paper presented at the Extended Abstracts of the 2018 CHI Conference on Human Factors in Computing Systems.

[10] R.,D.(2000).Interactive Installations For The Mind And Body.

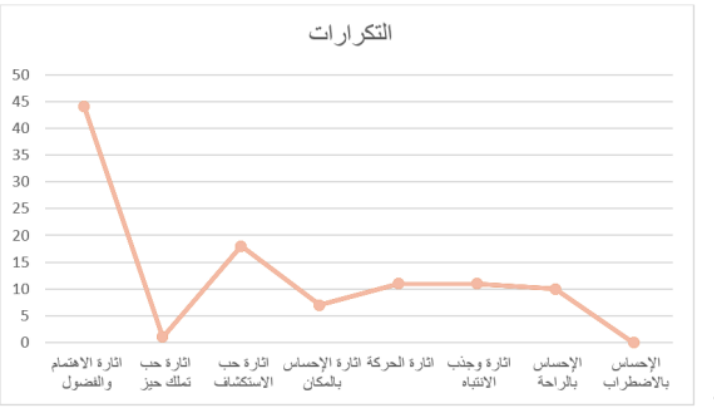

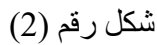

• اذا نظرنا الى الجدول رقم (5) نلاحظ ان اثارة الاهتمام

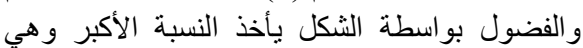
0.75 فمعظم الأمثلة تثير الاهتمام لدى الأشخاص

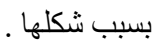

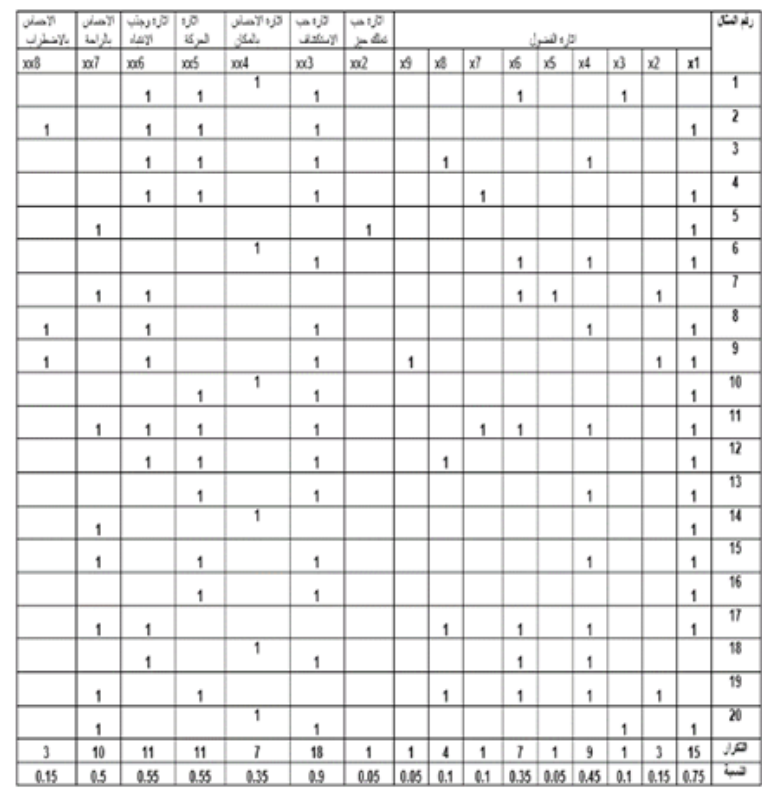

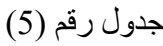

نلاحظ ان العينة رقم (4) أرضية Guildhall و العينة رقم (1) ساحة peter من المجموعة الاولى هما العينتان اللتان تحققان

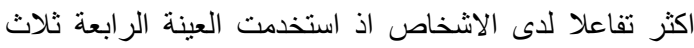

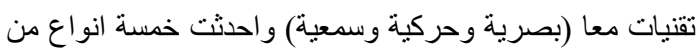

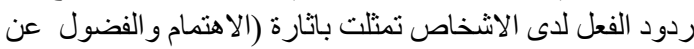

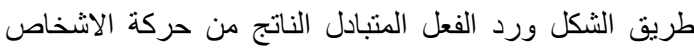

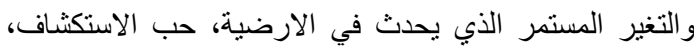

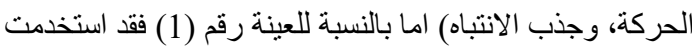

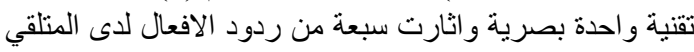

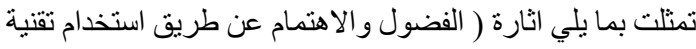

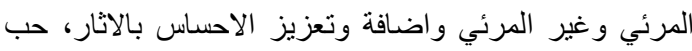

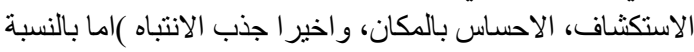

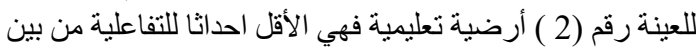

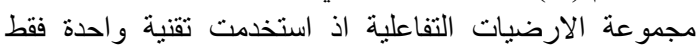
واحدثت 4 من ردود الفعل . مضيات الفغاعل 
[26] J. Bruges. (2013). Interactive Artwork Will Turn Your Shadow Into An Animation. Retreverd on 12/12/2020 : https://www.vice.com/ en /article /4xqg3b/artworkwill-turn-your-shadow-into-an-animation.

[27] P. JOHNSON. (2011) STRINGY ELLOW TUBE PLAYSCAPE BLANTON MUSEUM OF ART , HOUSTON TEXAS Retreverd on : Play-scapes.com

[28] https://www. albayan.ae /editors-choice/varity/201307-22-1.1927516 Retreverd on .2020/11/29،2013):

[29] J. Giannetto. (2019). 10 things you shouldn't miss at Chicago's Millennium Park Campus. Retreverd on 12/12/2020: https://www · choosechicago .com/blog/arts-culture-entertainment/10-things-youshouldnt-miss-at-chicagos-millennium-park-campus/

[30]. Retreverd on 20/12/2020 : https://www. youm7.com/story $\quad / 2020 / 9 \quad / 22 / 100$ \%D9\%85\%D9\%86\%D8 \%AD\%D9\% 88

[31] wordlesstechteam. (2012). THE WATERFALL SWING. Retreverd on 20/2/2020: https:// wordlesstech.com/waterfall-swing/

[32] A. Yoo. (2012). Color Changing Bubbles Tell Stories Through Shadows. Retreverd on 20/12/2020: https://mymodernmet.com/media-lucion-shadowballs/

[33] S. V. KARAARSLAN. (2018). APPLICATION OF AUGMENTED REALITY TECHNOLOGIES IN ARCHAEOLOGY

Retreverd on20/12/2020: (arkeotekno.com)
[11] A., D. A. A. R. (2013). The Effect of Artificial Lighting in the Nightscape Figuration of the Landscape University of Technology Baghdad

[12] Venkatesha,A.(2014).High school landscapes.

[13] E., R., \& Kenawy, I. (2016). Impact of outdoor landscape on students' social and Environmental behaviour. Paper presented at the The 2nd International Conference on Sustainability and the Future.

[14] M. Mahmoud Ahmed Shehata , I. M. M., Elham Rushdy Shata. (2018). Non-electronic interactive methods and their role in the interactive, advertising design. Architecture and Arts 11 (second part ).

[15] K. Krebel. (2013) Petar Zoranić Square and Šime Budinić Plaza / Kostrenčić-Krebel. Retreverd on 23/11/2020: https://www. archdaily.com 1478606/petar-zoranic-square-and-sime-budinicplaza-kostrencic-krebel?ad_medium=gallery

[16] EPA.2016 The floor is lava, and other optical illusion artworks pop up in South Korea. Retreverd on 20/11/2020 : https://www . bostonglobe .com /news/world/2016/10/27/the-floor-lava-and-otheroptical-illusion-artworks-pop-southkorea/qtkbY3qRDSBLdfG6Wyj1JJ/story.html

[17] FGTeam. (2016). Sensigom Delivers Versatile Range of Interactive Solutions for Indoor and Outdoor Play.Retreverd on 5/12/2020 https://www.fitnessgaming.com/news /schools/ sensigom-deliversversatile-range-of-interactive-solutions-for-indoorand-outdoor-play.html

[18] J. King. (2013). Song, dance and light in Southampton as artwork sends Guildhall square aglow. Retreverd on 6/11/2020: https:// www . culture24 .org.uk/art/art 457502

[19] K. Hosmer. (2013) Rows of Reflective Boxes Illustrate Portuguese Street Party. Retreverd on 2/10/2020https :// mymodernmet . com/fahr-estrutura-de-s-joao/

[20] G. Guido. (2019) Theory of Time, the installation revealed by its own shadow. Retreverd on 6/11/2020 : https:// www . collater.al/en/theory-of-time-dakuinstallation/

[21] c. brown. (2012). cloud made from 6,000 light bulbs by caitlind brown. Retreverd on 20/11/2020: https://www.designboom .com/ design/ cloud-madefrom-6000-light-bulbs-by-caitlind-brown/

[22] S. DAMODA. (2020) THE NAVIGATOR OF FORMATION DRONE LIGHT SHOW. Retreverd on 22/12/2020: http:// en. Dmduav . com/list-17.html

[23] D. Howarth. (2014, Les Astronautes fills Quebec passageway with swimming pool toys. Retreverd on $10 / 10 / 2020 \quad$ : https://www.dezeen. com/2014/10/13/ les-astronautes-delirious-fritespool-noodles-installation-quebec/

[24] A. Yoo. (2013,). Maze of Mirrors Mesmerizes Visitors Inside Sydney's Hyde Park. Retreverd on 16/11/2020: https://mymodernmet . com/art-and-about-sydneyfield/

[25] z. ong. (2017). NIKE's unlimited stadium in manila is the 'world's first LED running track'. Retreverd on 22/10/2020 : https://www. designboom.com /design/nike-unlimited-stadium-manila-worlds-firstled-running-track-05-08-2017/ 


\title{
Interactive Design in Urban Outdoor Spaces A Comparative Study of the Employment of Digital and Traditional Technologies
}

\author{
Suha Imad Aldobouni \\ suha.enp122@student.uomosul.edu.iq
}

\author{
Ahmed Yousif Al-Omary \\ ahmed.alomary@uomosul.edu.iq
}

Architecture Engineering Department, College of Engineering, University of Mosul

\begin{abstract}
Interactive design is a modern design approach that have gained a clear interest in the field of outdoor spaces. It depends on increasing elements of suspense, pleasure, learning, changing behavior and enriching the users' sensory experience and interaction through the five senses, to achieve an enjoyable experience and a good function that is easy to use. The research problem represented by the existence of traditional, passive and inactive urban spaces intended by few urban users. The research aims to explore employing digital technologies, formulas and methods of generating them for the purpose of applying them in external spaces, trying to revive urban outer spaces in contemporary cities. In this study, many examples of projects designed and implemented for external spaces that employ digital and traditional technologies have been analyzed and compared with each other, based on interaction criteria extracted from previous studies. Through the application of statistical tests, the results indicated that designs based on digital technologies generate relatively more interaction than those that rely on traditional techniques. The results indicated that optical technology is the most widely used technique in interactive outdoor spaces. The most effective interactive technologies on the recipient work through stimulating interest and curiosity.
\end{abstract}

Key words :

Interactive, sensory landscape, Urban outdoor spaces, interactive design, interactive Technique 